\title{
Majority decisions based on difference of votes ${ }^{\dagger}$
}

\author{
José Luis García-Lapresta* \& Bonifacio Llamazares \\ Universidad de Valladolid (Spain) \\ Dep. de Economía Aplicada (Matemáticas) \\ Facultad de Ciencias Económicas y Empresariales
}

\begin{abstract}
In this paper a class of voting procedures, located between simple and unanimous majorities, is introduced and characterized. Given two alternatives, the winning alternative is the one with a number of votes exceeding that obtained by the other in a previously fixed quantity. Moreover, a subclass of these voting procedures has been considered, by demanding additionally a number of votes greater than a previously fixed threshold. The main results of this paper are characterizations of these voting procedures through aggregation functions of fuzzy preferences associated with quasiarithmetic means and OWA operators.
\end{abstract}

\section{J.E.L. classification: D71.}

Keywords: Voting; Majorities; Fuzzy preferences; Quasiarithmetic means; OWA operators.

\section{Introduction}

When only two alternatives are in conflict, simple and absolute majorities are the most used voting procedures. In some cases, when the decision to take is very important, several qualified majorities, such as two thirds, are also used. Nevertheless, a wide class of decisions, of very different kind and importance, are taken with the same voting procedure.. In our opinion, according to the number of voters and the nature or transcendency of the decision, the voting procedure should be different. We note that some special majorities have been studied by Fishburn (1973, chapter 6) and Ferejohn - Grether (1974), among others.

\footnotetext{
${ }^{\dagger}$ The financial support of the Junta de Castilla y León (Consejería de Educación y Cultura, proyecto VA09/98) is gratefully acknowledged. The authors are grateful to an anonymous referee for valuable comments.

*Dep. de Economía Aplicada (Matemáticas). Facultad de Ciencias Económicas y Empresariales. Avda. Valle de Esgueva 6, 47011 Valladolid, Spain. Phone: $34+983+423330$. Fax: $34+983+423299$. E-mail: lapresta@cpd.uva.es.
} 
In this paper two classes of voting procedures are studied. Firstly we consider the difference of votes as the criterion to decide the winning alternative. After that, we also take into account a threshold of votes, which must be surpassed for an alternative to defeat. Both numbers, the difference and the threshold of votes, can previously be chosen in each decision problem in order to fix the desired support.

More specifically, we suppose $m$ voters, with $m \geq 3$, and two alternatives $x$ and $y$. In simple majority voting, $x$ defeats $y$ when the number of individuals who prefer $x$ to $y$ is greater than the number of individuals who prefer $y$ to $x$. Therefore, when there is high abstention, an alternative can be elected with very poor support. For example, we suppose a hundred voters; two of them prefer $x$ to $y$; one prefers $y$ to $x$ and the remaining ninety seven are indifferent. In this case, the simple majority chooses $x$ as the elected alternative. This choice seems inappropriate. For this reason, other kinds of majorities are used. The best known is absolute majority voting. In this case an alternative needs more than one half of the voters' support to be selected. But there are other qualified majorities, such as two thirds or three fourths majorities, where at least two thirds or three fourths of votes are needed. And unanimous majority, where the support of all the voters is necessary. Although these kinds of voting procedures solve the problem of abstention, they require a large number of votes. Consequently, there is a increasing loss of decisivity.

To avoid in some way the two problems mentioned, power of abstention and loss of decisivity, in this paper we have introduced a class of voting procedures based on difference of votes, the $M_{k}$ majorities (similar classes were noted by Fishburn (1973, p. 18) and Saari (1990, pp. 122-123)). For $M_{k}, x$ is collectively preferred to $y$ when the number of individuals who prefer $x$ to $y$ exceeds the number of individuals who prefer $y$ to $x$ by at least a fixed integer $k$ between 0 and $m-1$. We note that $M_{k}$ majorities are found between simple majority, in the extreme case of $k=0$, and unanimous majority, in the other extreme case, $k=m-1$.

Moreover, we have introduced a modification of the previous class of voting procedures, the $M_{k}^{l}$ majorities, where $x$ is collectively preferred to $y$ when the number of voters who prefer $x$ to $y$ simultanously exceeds the threshold $l$ and the number of voters who prefer $y$ to $x$ in the quantity $k$.

The two classes of voting procedures, $M_{k}$ and $M_{k}^{l}$ majorities, are neutral, anonymous, monotonic and unanimous discrete aggregation functions. In both cases we will suppose that voters have, or have to show, crisp preferences. $M_{k}$ and $M_{k}^{l}$ majorities can be adapted in a more appropiate way than simple and absolute majorities, to a wide range of realistic situations. It is only necessary to choose the more convenient values of the parameters $k$ and $l$, according to each case.

In this paper we have proven several results where the above voting procedures are generated by two classes of aggregation functions: quasiarithmetic means and OWA operators. By means of these aggregation functions it is possible to extend $M_{k}$ and $M_{k}^{l}$ majorities to fuzzy case maintaining anonymity, neutrality, monotonicity and unanimity. This viewpoint for extending 
voting rules from crisp to fuzzy preferences is different to the one used by some authors, who adapt the axioms of the crisp case to the fuzzy case (see, for example, Marchant (1996) and (2000)).

Quasiarithmetic means were characterized by Kolmogoroff (1930) and Nagumo (1930). In Bullen - Mitrinović - Vasić (1988) there is an exhaustive study on means (chapters IV and VI are devoted to quasiarithmetic meaans). On results about quasiarithmetic means as aggregation rules of fuzzy preferences, see Ovchinnikov (1990) and García-Lapresta - Llamazares (2000), among others. OWA ("Ordered Weighted Averaging") operators were introduced by Yager (1988); a wide study can be found in Yager et al. (1997). On aggregation functions, see Dubois - Prade (1985), Yager (1991), Fodor - Roubens (1994) and Grabisch (1996), among other contributions.

The organization of the paper is as follows. In Section 2 we introduce aggregation functions. They define the collective preference between alternatives as an outcome of individual preferences, fuzzy or crisp. In both cases, we consider some properties of aggregation functions: anonymity, neutrality (in the sense of May, 1952), monotonicity and unanimity. In Section 3 the two voting procedures studied in the paper, $M_{k}$ and $M_{k}^{l}$ majorities, are formally introduced. Sections 4 and 5 are devoted to the aggregation functions corresponding to quasiarithmetic means and OWA operators, respectively. These sections contain the main results of the paper.

\section{Aggregation functions}

Individuals who prefer one alternative to other, can prefer it with different levels of intensity. Fuzzy preferences reflect this information, evaluating the levels of preference intensity between 0 and 1 . So, with $r_{i} \in[0,1]$ we denote the intensity with which voter $i$ prefers $x$ to $y$. Under this assumption it is usual to suppose that $1-r_{i}$ is the intensity with which voter $i$ prefers $y$ to $x$ (see Bezdek et al. (1978), Nurmi (1981), Tanino (1984) and Nakamura (1992), among others). If $r_{i} \in\left\{0, \frac{1}{2}, 1\right\}$, we say that voter $i$ has crisp preferences. In this case, we understand that $r_{i}$ is 1,0 or $\frac{1}{2}$, if voter $i$ prefers $x$ to $y$, prefers $y$ to $x$ or is indifferent between the two alternatives, respectively. For more details, see García-Lapresta - Llamazares (2000).

A profile of preferences is a vector $\mathbf{r}=\left(r_{1}, \ldots, r_{m}\right)$ which describes the voters' preferences of $x$ to $y$. Obviously, $\left(1-r_{1}, \ldots, 1-r_{m}\right)$ shows the voters' preferences of $y$ to $x$.

Definition 1. An aggregation function is a mapping $F:[0,1]^{m} \longrightarrow[0,1]$. Given a profile of preferences $\mathbf{r}=\left(r_{1}, \ldots, r_{m}\right) \in[0,1]^{m}, F(\mathbf{r})$ is the intensity with which $x$ is collectively preferred to $y$. A discrete aggregation function (DAF) is a mapping $F:\left\{0, \frac{1}{2}, 1\right\}^{m} \longrightarrow\left\{0, \frac{1}{2}, 1\right\}$. Given a profile of crisp preferences $\mathbf{r}=\left(r_{1}, \ldots, r_{m}\right) \in\left\{0, \frac{1}{2}, 1\right\}^{m}, F(\mathbf{r})$ shows us if $x$ is collectively preferred to $y, y$ is collectively preferred to $x$, or $x$ and $y$ are collectively indifferent, according 
to whether $F(\mathbf{r})$ is 1,0 or $\frac{1}{2}$, respectively.

Now we introduce several properties of the aggregation functions: anonymity, neutrality, monotonicity and unanimity. Anonymity means that collective intensity of preference depends on only the set of individual intensity of preferences, but not on which individuals have these preferences. Neutrality means that if everyone reverses their preference between $x$ and $y$, then the collective preference is also reversed. Thus, neutrality assures an egalitarian treatment to the alternatives: the collective intensity of preference between two alternatives does not depends on the label of the alternatives. Monotonicity means that collective intensity of preference does not decrease if no individual intensity decreases. And unanimity means that collective intensity of preference coincides with individual intensities when these are the same.

Definition 2. Let $F$ be an aggregation function or a DAF.

1. $F$ is anonymous if and only if for all profile $\mathbf{r}=\left(r_{1}, \ldots, r_{m}\right)$ and for all permutation $\sigma$ of $\{1, \ldots, m\}$ the following holds

$$
F\left(r_{\sigma(1)}, \ldots, r_{\sigma(m)}\right)=F(\mathbf{r}) .
$$

2. $F$ is neutral if and only if for all profile $\left(r_{1}, \ldots, r_{m}\right)$ the following holds

$$
F\left(1-r_{1}, \ldots, 1-r_{m}\right)=1-F\left(r_{1}, \ldots, r_{m}\right) .
$$

3. $F$ is monotonic if and only if for all pair of profiles $\left(r_{1}, \ldots, r_{m}\right)$ and $\left(r_{1}^{\prime}, \ldots, r_{m}^{\prime}\right)$ the following holds

$$
\forall i \in\{1, \ldots, m\} \quad r_{i}^{\prime} \geq r_{i} \Rightarrow F\left(r_{1}^{\prime}, \ldots, r_{m}^{\prime}\right) \geq F\left(r_{1}, \ldots, r_{m}\right) .
$$

4. $F$ is unanimous if and only if for all $r$ the following holds $F(r, \ldots, r)=r$.

Now some consequences of the previous properties are obtained. The cardinal of a set will be denoted by means of \#.

Remark 1. If $F:\left\{0, \frac{1}{2}, 1\right\}^{m} \longrightarrow\left\{0, \frac{1}{2}, 1\right\}$ is an anonymous DAF, then $F(\mathbf{r})$ depends on only the number of $1, \frac{1}{2}$ and 0 . Given a profile $\left(r_{1}, \ldots, r_{m}\right)$, if we consider:

1. $m_{1}=\#\left\{i \mid r_{i}=1\right\}$, the number of voters who prefer $x$ to $y$,

2. $m_{2}=\#\left\{i \mid r_{i}=\frac{1}{2}\right\}$, the number of voters who are indifferent between $x$ and $y$,

3. $m_{3}=\#\left\{i \mid r_{i}=0\right\}$, the number of voters who prefer $y$ to $x$,

then $m_{1}+m_{2}+m_{3}=m$. 
By Remark 1, every anonymous DAF can be represented by a mapping over the triples $\left(m_{1}, m_{2}, m_{3}\right) \in\{0,1, \ldots, m\}^{3}$ such that $m_{1}+m_{2}+m_{3}=m$.

Definition 3. Let $F:\left\{0, \frac{1}{2}, 1\right\}^{m} \longrightarrow\left\{0, \frac{1}{2}, 1\right\}$ be an anonymous DAF. We define the mapping $f: \mathcal{M} \longrightarrow\left\{0, \frac{1}{2}, 1\right\}$, where

$$
\mathcal{M}=\left\{\left(m_{1}, m_{2}, m_{3}\right) \in\{0,1, \ldots, m\}^{3} \mid m_{1}+m_{2}+m_{3}=m\right\}
$$

and

$$
f\left(m_{1}, m_{2}, m_{3}\right)=F\left(1, \stackrel{\left(m_{1}\right)}{\cdot}, 1, \frac{1}{2}, \stackrel{\left(m_{2}\right)}{\cdots}, \frac{1}{2}, 0,\left(\stackrel{m_{3}}{*}\right), 0\right) .
$$

We say that $F$ is represented by $f$.

Moreover, we define

$$
C(f)=\left\{\left(m_{1}, m_{2}, m_{3}\right) \in \mathcal{M} \mid f\left(m_{1}, m_{2}, m_{3}\right)=1\right\} .
$$

Remark 2. If $F:[0,1]^{m} \longrightarrow[0,1]$ is an anonymous aggregation function, then the restriction of $F$ to profiles of crisp preferences,

$$
\left.F\right|_{\left\{0, \frac{1}{2}, 1\right\}^{m}}:\left\{0, \frac{1}{2}, 1\right\}^{m} \longrightarrow[0,1]
$$

is determined, as in the case of the anonymous DAF, by the number of $1, \frac{1}{2}$ and 0 . Hence, $\left.F\right|_{\left\{0, \frac{1}{2}, 1\right\}^{m}}$ can be represented by a mapping $f: \mathcal{M} \longrightarrow[0,1]$, where

$$
f\left(m_{1}, m_{2}, m_{3}\right)=F\left(1, \stackrel{\left(m_{1}\right)}{\cdots}, 1, \frac{1}{2}, \stackrel{\left(m_{2}\right)}{\cdots}, \frac{1}{2}, 0, \stackrel{\left(m_{3}\right)}{\cdots}, 0\right) .
$$

Remark 3. Let $F:\left\{0, \frac{1}{2}, 1\right\}^{m} \longrightarrow\left\{0, \frac{1}{2}, 1\right\}$ be an anonymous DAF represented by the mapping $f: \mathcal{M} \longrightarrow\left\{0, \frac{1}{2}, 1\right\}$. Then $F$ is neutral if and only if for all $\left(m_{1}, m_{2}, m_{3}\right) \in \mathcal{M}$ it is satisfied $f\left(m_{1}, m_{2}, m_{3}\right)+f\left(m_{3}, m_{2}, m_{1}\right)=1$. In this case $F$ is characterized by the set $C(f)$, since $f\left(m_{1}, m_{2}, m_{3}\right)=0$ is equivalent to $\left(m_{3}, m_{2}, m_{1}\right) \in C(f)$, and $f\left(m_{1}, m_{2}, m_{3}\right)=\frac{1}{2}$ is equivalent to $\left(m_{1}, m_{2}, m_{3}\right) \notin C(f)$ and $\left(m_{3}, m_{2}, m_{1}\right) \notin C(f)$.

Remark 4. If $F$ is a monotonic and unanimous aggregation function or DAF, then

$$
\min \left(r_{1}, \ldots, r_{m}\right) \leq F\left(r_{1}, \ldots, r_{m}\right) \leq \max \left(r_{1}, \ldots, r_{m}\right),
$$

for all profile $\left(r_{1}, \ldots, r_{m}\right)$. This property is satisfied by all the DAF studied in the paper. It guarantees that collective intensity of preference is located between minimum and maximum individual intensities of preference. It has been widely used in the literature (in Fodor - Roubens (1994, p. 108) this property is called compensative). 
When voters have crisp preferences, every aggregation function $F:[0,1]^{m} \longrightarrow[0,1]$ can be restricted to profiles of crisp preferences, $\left.F\right|_{\left\{0, \frac{1}{2}, 1\right\}^{m}}:\left\{0, \frac{1}{2}, 1\right\}^{m} \longrightarrow[0,1]$. If we wish to obtain a DAF from $F$, then it will be necessary for collective intensities of preference to be $0, \frac{1}{2}$ or 1 . Now we present a way to generate a DAF from an aggregation function when voters have, or have to show, crisp preferences.

Definition 4. Given an aggregation function $F:[0,1]^{m} \longrightarrow[0,1]$ and $\alpha \in\left[\frac{1}{2}, 1\right)$, we define the $\alpha$-DAF associated with $F$ as the DAF $F_{\alpha}:\left\{0, \frac{1}{2}, 1\right\}^{m} \longrightarrow\left\{0, \frac{1}{2}, 1\right\}$ defined by

$$
F_{\alpha}\left(r_{1}, \ldots, r_{m}\right)= \begin{cases}1, & \text { if } F\left(r_{1}, \ldots, r_{m}\right)>\alpha \\ \frac{1}{2}, & \text { if } 1-\alpha \leq F\left(r_{1}, \ldots, r_{m}\right) \leq \alpha \\ 0, & \text { if } F\left(r_{1}, \ldots, r_{m}\right)<1-\alpha\end{cases}
$$

Now we present the following proposition whose proof is straightforward.

Proposition 1. Given an aggregation function $F:[0,1]^{m} \longrightarrow[0,1]$, for all $\alpha \in\left[\frac{1}{2}, 1\right)$ the following statements hold:

1. If $F$ is anonymous, then $F_{\alpha}$ is also anonymous.

2. If $F$ is neutral, then $F_{\alpha}$ is also neutral.

3. If $F$ is monotonic, then $F_{\alpha}$ is also monotonic.

4. If $F$ is unanimous, then $F_{\alpha}$ is also unanimous.

Remark 5. Let $F:[0,1]^{m} \longrightarrow[0,1]$ be an aggregation function and $\alpha \in\left[\frac{1}{2}, 1\right)$. If $F$ is anonymous, by Remark $5, F_{\alpha}$ is an anonymous DAF and, consequently, it can be represented by a mapping $f_{\alpha}: \mathcal{M} \longrightarrow\left\{0, \frac{1}{2}, 1\right\}$, where

$$
f_{\alpha}\left(m_{1}, m_{2}, m_{3}\right)=F_{\alpha}\left(1, \stackrel{\left(m_{1}\right)}{\cdots}, 1, \frac{1}{2}, \stackrel{\left(m_{2}\right)}{\cdot}, \frac{1}{2}, 0, \stackrel{\left(m_{3}\right)}{\cdot}, 0\right) .
$$

\section{$3 \quad M_{k}$ and $M_{k}^{l}$ majorities}

Now we introduce the two classes of DAF corresponding to the $M_{k}$ and $M_{k}^{l}$ majorities studied in this paper. 
Definition 5. Given $k \in\{0,1, \ldots, m-1\}$, we define the $M_{k}$ majority as the anonymous DAF defined by

$$
f\left(m_{1}, m_{2}, m_{3}\right)= \begin{cases}1, & \text { if } m_{1}>m_{3}+k \\ \frac{1}{2}, & \text { if }\left|m_{1}-m_{3}\right| \leq k \\ 0, & \text { if } m_{3}>m_{1}+k\end{cases}
$$

In other words, $x$ is collectively preferred to $y$ by $M_{k}$ when the number of voters who prefer $x$ to $y$ exceeds those who prefer $y$ to $x$ in the prefixed number of votes $k$. Analogously, $y$ is collectively preferred to $x$ when the number of voters who prefer $y$ to $x$ exceeds those who prefer $x$ to $y$ in $k$ votes. Alternatives $x$ and $y$ are collectively indifferent if the absolute difference between the number of votes obtained by each one of them does not exceed $k$.

We note that $M_{k}$ is neutral, monotonic and unanimous.

Remark 6. Simple and unanimous majorities are particular cases of $M_{k}$ majorities.

1. If $k=0$, we obtain simple majority.

2. If $k=m-1, x$ is collectively preferred to $y$ if and only if all the individuals prefer $x$ to $y$.

Remark 7. When different values of $k$ are taken, different $M_{k}$ majorities are obtained, i.e., if $k, k^{\prime} \in\{0,1, \ldots, m-1\}$, then $M_{k}=M_{k^{\prime}} \Leftrightarrow k=k^{\prime}$.

It is possible to define the class of $M_{k}$ majorities by considering percentages instead number of individuals, in a similar way to Saari $\left(1994\right.$, p. 30). In this case, $M_{k}$ majorities do not depend on the total number of individuals, $m$.

Remark 8. Given $\rho \in[0,1)$ and

$$
\mathcal{S}=\left\{\left(x_{1}, x_{2}, x_{3}\right) \in \mathbb{Q}^{3} \mid x_{1} \geq 0, x_{2} \geq 0, x_{3} \geq 0, x_{1}+x_{2}+x_{3}=1\right\},
$$

we can define on $\mathcal{S}$ a majority by means of the mapping $f: \mathcal{S} \longrightarrow\left\{0, \frac{1}{2}, 1\right\}$, where

$$
f\left(x_{1}, x_{2}, x_{3}\right)= \begin{cases}1, & \text { if } x_{1}>x_{3}+\rho \\ \frac{1}{2}, & \text { if }\left|x_{1}-x_{3}\right| \leq \rho \\ 0, & \text { if } x_{3}>x_{1}+\rho .\end{cases}
$$


This majority corresponds to $M_{k}$ majority for $\rho=\frac{k}{m}$. We note that simple majority is obtained for $\rho=0$. However, it is not possible to find a value for $\rho$ in order to obtain unanimous majority.

Now we introduce $M_{k}^{l}$ majorities, a subclass of $M_{k}$ majorities. Given $a \in \mathbb{N}$, with $[a]$ we denote the integer part of $a$, i.e., the largest integer smaller than or equal to $a$.

Definition 6. Given $k \in\{0,1, \ldots, m-4\}$ and $l \in\left\{k+1, \ldots,\left[\frac{m+k}{2}\right]-1\right\}$, we define the $M_{k}^{l}$ majority as the anonymous DAF defined by

$$
f\left(m_{1}, m_{2}, m_{3}\right)= \begin{cases}1, & \text { if } m_{1}>\max \left(m_{3}+k, l\right), \\ \frac{1}{2}, & \text { if }\left|m_{1}-m_{3}\right| \leq k \text { or } \max \left(m_{1}, m_{3}\right) \leq l, \\ 0, & \text { if } m_{3}>\max \left(m_{1}+k, l\right) .\end{cases}
$$

In other words, $x$ is collectively preferred to $y$ when the number of voters who prefer $x$ to $y$ simultaneously exceeds the threshold $l$ and the number of voters who prefer $y$ to $x$ in the quantity $k$. Analogously, $y$ is collectively preferred to $x$ when the number of voters who prefer $y$ to $x$ simultaneously exceeds the threshold $l$ and the number of voters who prefer $x$ to $y$ in the quantity $k$. Alternatives $x$ and $y$ will be collectively indifferent if the absolute difference between the votes obtained by each one of them does not exceed $k$ or if no alternative exceeds the number of votes fixed by the threshold $l$.

We note that $M_{k}^{l}$ is neutral, monotonic and unanimous.

Remark 9. In Definition 6, the parameters $k$ and $l$ take their values in the corresponding sets so that the conditions $m_{1}>m_{3}+k$ and $m_{1}>l$ will both be effective. The restriction $l \geq k+1$ is necessary to guarantee that the condition $m_{1}>l$ will not be redundant. On the other hand, $m_{1}>m_{3}+k$ is not redundant if there exists a profile of crisp preferences satisfying $m_{1}>l$, but not $m_{1}>m_{3}+k$. In order to find the maximum possible value of $l$, we consider the minimum possible values of $m_{1}$ and $m_{3}$ that satisfy the conditions $m_{1}>l$ and $m_{1} \leq m_{3}+k$. These values are $m_{1}=l+1$ and $m_{3}=m_{1}-k=l+1-k$. If we consider the restriction $m_{1}+m_{3} \leq m$, the maximum value of $l$ is the integer solution of the equation $(l+1)+(l+1-k)=m$, i.e., $l=\left[\frac{m+k}{2}\right]-1$. Moreover, since $k+1 \leq l$, the maximum possible value of $k$ is obtained taking $l=k+1$ in $(l+1)+(l+1-k)=m$, i.e., $k=m-4$.

Remark 10. When different values of $k$ and $l$ are taken, different $M_{k}^{l}$ majorities are obtained, i.e., if $k, k^{\prime} \in\{0,1, \ldots, m-4\}$ and $l, l^{\prime} \in\left\{k+1, \ldots,\left[\frac{m+k}{2}\right]-1\right\}$, then $M_{k}^{l}=M_{k^{\prime}}^{l^{\prime}} \Leftrightarrow k=k^{\prime}$ and $l=l^{\prime}$.

Analogously to $M_{k}$ majorities, it is possible to define the class of $M_{k}^{l}$ majorities when we only consider percentage of individuals. 
Remark 11. Given $\rho \in[0,1), \theta \in\left(\rho, \frac{1+\rho}{2}\right)$ and

$$
\mathcal{S}=\left\{\left(x_{1}, x_{2}, x_{3}\right) \in \mathbb{Q}^{3} \mid x_{1} \geq 0, x_{2} \geq 0, x_{3} \geq 0, x_{1}+x_{2}+x_{3}=1\right\},
$$

we can define on $\mathcal{S}$ a majority by means of the mapping $f: \mathcal{S} \longrightarrow\left\{0, \frac{1}{2}, 1\right\}$, where

$$
f\left(x_{1}, x_{2}, x_{3}\right)= \begin{cases}1, & \text { if } x_{1}>\max \left(x_{3}+\rho, \theta\right) \\ \frac{1}{2}, & \text { if }\left|x_{1}-x_{3}\right| \leq \rho \text { or } \max \left(x_{1}, x_{3}\right) \leq \theta \\ 0, & \text { if } x_{3}>\max \left(x_{1}+\rho, \theta\right)\end{cases}
$$

This majority corresponds with $M_{k}^{l}$ majority for $\rho=\frac{k}{m}$ and $\theta=\frac{l}{m}$.

\section{Quasiarithmetic means}

In this section we study a class of aggregation functions widely used in the literature: quasiarithmetic means. Furthermore, we prove that $M_{k}$ majorities are generated through neutral quasiarithmetic means.

Definition 7. A mapping $\varphi:[0,1] \longrightarrow[0,1]$ is an order automorphism if and only if $\varphi$ is bijective and increasing.

Now we show some properties of order automorphisms (see García-Lapresta - Llamazares (2000, pp. 684-685)).

Proposition 2. For every order automorphism $\varphi:[0,1] \longrightarrow[0,1]$ the following statements hold:

1. $\varphi$ is strictly increasing.

2. $\varphi^{-1}$ is an order automorphism.

3. $\varphi(0)=0$ and $\varphi(1)=1$.

4. $\varphi$ is continuous.

Definition 8. An order automorphism $\varphi:[0,1] \longrightarrow[0,1]$ is neutral if and only if the following holds $\varphi(a)+\varphi(1-a)=1$ for all $a \in[0,1]$.

Obviously, if $\varphi$ is a neutral order automorphism, then $\varphi\left(\frac{1}{2}\right)=\frac{1}{2}$ and $\varphi^{-1}$ is also neutral. 
Definition 9. Given an order automorphism $\varphi:[0,1] \longrightarrow[0,1]$, the quasiarithmetic mean associated with $\varphi$ is the aggregation function $F^{\varphi}$ defined by

$$
F^{\varphi}\left(r_{1}, \ldots, r_{m}\right)=\varphi^{-1}\left(\frac{\varphi\left(r_{1}\right)+\cdots+\varphi\left(r_{m}\right)}{m}\right) .
$$

Simple examples of quasiarithmetic means are

$$
F^{\varphi}\left(r_{1}, \ldots, r_{m}\right)= \begin{cases}\frac{r_{1}+\cdots+r_{m}}{m}, & \text { when } \varphi(a)=a, \\ \sqrt{\frac{r_{1}^{2}+\cdots+r_{m}^{2}}{m}}, & \text { when } \varphi(a)=a^{2}, \\ \left(\frac{r_{1}^{p}+\cdots+r_{m}^{p}}{m}\right)^{\frac{1}{p}}, & \text { when } \varphi(a)=a^{p} \quad(p>0) .\end{cases}
$$

We note that Ovchinnikov (1990, Th. 5.1) characterizes the quasiarithmetic means, when individuals have crisp preferences, by means of some properties of fuzzy aggregation; the restrictions of these properties on the ordinary case are similar to those given by May (1952) for simple majority voting.

Now we establish that every quasiarithmetic mean is anonymous, monotonic and unanimous. Moreover, we give a necessary and sufficient condition so that it will be neutral.

Proposition 3. For every order automorphism $\varphi:[0,1] \longrightarrow[0,1]$ the following statements hold:

1. $F^{\varphi}$ is anonymous, monotonic and unanimous.

2. $F^{\varphi}$ is neutral $\Leftrightarrow \varphi$ is neutral.

Proof.

1. Obvious.

2. $\Rightarrow$ ) It is sufficient to prove that $\varphi^{-1}$ is neutral. For each $n \in \mathbb{N}$, we define the set

$$
\mathcal{C}_{n}=\left\{\frac{p}{m^{n}} \mid p \in\left\{0,1, \ldots, m^{n}\right\}\right\} .
$$

We prove by induction on $n$ that $\varphi^{-1}$ is neutral in the points of the sets $\mathcal{C}_{n}$. For $n=1$, let $p \in\{0,1, \ldots, m\}$ and $\left(r_{1}, \ldots, r_{m}\right) \in[0,1]^{m}$ such that

$$
r_{i}= \begin{cases}1, & \text { if } i \in\{1, \ldots, p\}, \\ 0, & \text { if } i \in\{p+1, \ldots, m\} .\end{cases}
$$


Then, we have

$$
F^{\varphi}\left(r_{1}, \ldots, r_{m}\right)=\varphi^{-1}\left(\frac{1}{m}\left(\sum_{i=1}^{p} \varphi(1)+\sum_{i=p+1}^{m} \varphi(0)\right)\right)=\varphi^{-1}\left(\frac{p}{m}\right) .
$$

On the other hand,

$$
\begin{aligned}
& F^{\varphi}\left(r_{1}, \ldots, r_{m}\right)=1-F^{\varphi}\left(1-r_{1}, \ldots, 1-r_{m}\right)= \\
& =1-\varphi^{-1}\left(\frac{1}{m}\left(\sum_{i=1}^{p} \varphi(0)+\sum_{i=p+1}^{m} \varphi(1)\right)\right)= \\
& =1-\varphi^{-1}\left(\frac{m-p}{m}\right)=1-\varphi^{-1}\left(1-\frac{p}{m}\right) .
\end{aligned}
$$

Hence,

$$
\varphi^{-1}\left(1-\frac{p}{m}\right)=1-\varphi^{-1}\left(\frac{p}{m}\right)
$$

Suppose, by hypothesis of induction, that $\varphi^{-1}$ is neutral in the points of $\mathcal{C}_{k}$. Now we prove that $\varphi^{-1}$ is also neutral in the points of $\mathcal{C}_{k+1}$. If $p \in\left\{0,1, \ldots, m^{k+1}\right\}$, then there exist $p_{0} \in\{0,1, \ldots, m\}$ and $p_{1} \in\left\{0,1, \ldots, m^{k}-1\right\}$ such that $p=p_{0} m^{k}+p_{1}$. Consider $\left(r_{1}, \ldots, r_{m}\right) \in[0,1]^{m}$ such that

$$
r_{i}= \begin{cases}1, & \text { if } i \in\left\{1, \ldots, p_{0}\right\}, \\ \varphi^{-1}\left(\frac{p_{1}}{m^{k}}\right), & \text { if } i=p_{0}+1, \\ 0, & \text { if } i \in\left\{p_{0}+2, \ldots, m\right\} .\end{cases}
$$

Then, we have

$$
\begin{aligned}
& F^{\varphi}\left(r_{1}, \ldots, r_{m}\right)=\varphi^{-1}\left(\frac{1}{m}\left(\sum_{i=1}^{p_{0}} \varphi(1)+\varphi\left(\varphi^{-1}\left(\frac{p_{1}}{m^{k}}\right)\right)+\sum_{i=p_{0}+2}^{m} \varphi(0)\right)\right)= \\
& =\varphi^{-1}\left(\frac{1}{m}\left(p_{0}+\frac{p_{1}}{m^{k}}\right)\right)=\varphi^{-1}\left(\frac{p}{m^{k+1}}\right) .
\end{aligned}
$$

On the other hand,

$$
\begin{aligned}
& F^{\varphi}\left(r_{1}, \ldots, r_{m}\right)=1-F^{\varphi}\left(1-r_{1}, \ldots, 1-r_{m}\right)= \\
& =1-\varphi^{-1}\left(\frac{1}{m}\left(\sum_{i=1}^{p_{0}} \varphi(0)+\varphi\left(1-\varphi^{-1}\left(\frac{p_{1}}{m^{k}}\right)\right)+\sum_{i=p_{0}+2}^{m} \varphi(1)\right)\right)= \\
& =1-\varphi^{-1}\left(\frac{1}{m}\left(m-\left(p_{0}+1\right)+1-\frac{p_{1}}{m^{k}}\right)\right)= \\
& =1-\varphi^{-1}\left(\frac{1}{m}\left(m-\frac{p}{m^{k}}\right)\right)=1-\varphi^{-1}\left(1-\frac{p}{m^{k+1}}\right) .
\end{aligned}
$$


Hence,

$$
\varphi^{-1}\left(1-\frac{p}{m^{k+1}}\right)=1-\varphi^{-1}\left(\frac{p}{m^{k+1}}\right)
$$

Now we justify that $\mathcal{C}=\bigcup_{n=1}^{\infty} \mathcal{C}_{n}$ is dense in $[0,1]$. It is sufficient to prove that for every $r \in[0,1]-\mathcal{C}$ there exists an element of $\mathcal{C}$ whose distance to $r$ is less than a given $\varepsilon>0$. Let $n \in \mathbb{N}$ such that $\frac{1}{m^{n}}<\varepsilon$. Then, there exists $p \in\left\{0,1, \ldots, m^{n}-1\right\}$ such that $\frac{p}{m^{n}}<r<\frac{p+1}{m^{n}}$; so, we have $r-\frac{p}{m^{n}}<\varepsilon$.

Finally, we shall show that $\varphi^{-1}$ is neutral in every point of $[0,1]$. Given $r \in[0,1]$, there exists a sequence of points of $\mathcal{C},\left\{r_{n}\right\}_{n=1}^{\infty}$, which converges to $r$. Then, $\lim _{n \rightarrow \infty}\left(1-r_{n}\right)=1-r$ and, by the continuity of $\varphi^{-1}$, we have

$$
\begin{aligned}
& \varphi^{-1}(1-r)=\lim _{n \rightarrow \infty} \varphi^{-1}\left(1-r_{n}\right)=\lim _{n \rightarrow \infty}\left(1-\varphi^{-1}\left(r_{n}\right)\right)= \\
& =1-\lim _{n \rightarrow \infty} \varphi^{-1}\left(r_{n}\right)=1-\varphi^{-1}(r) .
\end{aligned}
$$

$\Leftarrow)$ Since $\varphi^{-1}$ is neutral, for every $\left(r_{1}, \ldots, r_{m}\right) \in[0,1]^{m}$ we have

$$
\begin{aligned}
& F^{\varphi}\left(1-r_{1}, \ldots, 1-r_{m}\right)=\varphi^{-1}\left(\frac{\varphi\left(1-r_{1}\right)+\cdots+\varphi\left(1-r_{m}\right)}{m}\right)= \\
& =\varphi^{-1}\left(1-\frac{\varphi\left(r_{1}\right)+\cdots+\varphi\left(r_{m}\right)}{m}\right)=1-\varphi^{-1}\left(\frac{\varphi\left(r_{1}\right)+\cdots+\varphi\left(r_{m}\right)}{m}\right)= \\
& =1-F^{\varphi}\left(r_{1}, \ldots, r_{m}\right) .
\end{aligned}
$$

Remark 12. For every order automorphism $\varphi:[0,1] \longrightarrow[0,1]$ and every $\alpha \in\left[\frac{1}{2}, 1\right)$, the anonymous DAF $F_{\alpha}^{\varphi}$ is represented by $f_{\alpha}^{\varphi}: \mathcal{M} \longrightarrow\left\{0, \frac{1}{2}, 1\right\}$, where

$$
f_{\alpha}^{\varphi}\left(m_{1}, m_{2}, m_{3}\right)=\left\{\begin{array}{l}
1, \quad \text { if } \varphi^{-1}\left(\frac{1}{m}\left(m_{1}+\frac{m_{2}}{2}\right)\right)>\alpha, \\
\frac{1}{2}, \quad \text { if } 1-\alpha \leq \varphi^{-1}\left(\frac{1}{m}\left(m_{1}+\frac{m_{2}}{2}\right)\right) \leq \alpha, \\
0, \quad \text { if } \varphi^{-1}\left(\frac{1}{m}\left(m_{1}+\frac{m_{2}}{2}\right)\right)<1-\alpha .
\end{array}\right.
$$

Moreover, according to Propositions 3 and 1, if $\varphi$ is neutral, then $F_{\alpha}^{\varphi}$ is a neutral DAF.

Now we establish that every $\alpha$-DAF associated with a neutral quasiarithmetic mean is a $M_{k}$ majority. 
Proposition 4. If $\varphi:[0,1] \longrightarrow[0,1]$ is a neutral order automorphism and $\alpha \in\left[\frac{1}{2}, 1\right)$, then $F_{\alpha}^{\varphi}=M_{[m(2 \varphi(\alpha)-1)]}$.

Proof. By Remark 12, for every $\left(m_{1}, m_{2}, m_{3}\right) \in \mathcal{M}$, we have

$$
\begin{aligned}
& f_{\alpha}^{\varphi}\left(m_{1}, m_{2}, m_{3}\right)=1 \Leftrightarrow \varphi^{-1}\left(\frac{1}{m}\left(m_{1}+\frac{m_{2}}{2}\right)\right)>\alpha \Leftrightarrow 2 m_{1}+m_{2}>2 m \varphi(\alpha) \Leftrightarrow \\
& \Leftrightarrow m_{1}>m_{3}+m(2 \varphi(\alpha)-1) \Leftrightarrow m_{1}>m_{3}+[m(2 \varphi(\alpha)-1)] .
\end{aligned}
$$

In the following theorem we justify that, for every $M_{k}$ majority and every neutral order automorphism $\varphi$, there exists some $\alpha \in\left[\frac{1}{2}, 1\right)$ such that $M_{k}$ is the $\alpha$-DAF associated with the quasiarithmetic mean defined by $\varphi$.

Theorem 1. If $\varphi:[0,1] \longrightarrow[0,1]$ is a neutral order automorphism, $k \in\{0, \ldots, m-1\}$ and $\alpha \in\left[\frac{1}{2}, 1\right)$, then

$$
M_{k}=F_{\alpha}^{\varphi} \Leftrightarrow \varphi^{-1}\left(\frac{m+k}{2 m}\right) \leq \alpha<\varphi^{-1}\left(\frac{m+k+1}{2 m}\right) .
$$

Proof. By Proposition 4 and Remark 7, we have

$$
\begin{aligned}
& M_{k}=F_{\alpha}^{\varphi} \Leftrightarrow M_{k}=M_{[m(2 \varphi(\alpha)-1)]} \Leftrightarrow k=[m(2 \varphi(\alpha)-1)] \Leftrightarrow k \leq 2 m \varphi(\alpha)-m<k+1 \Leftrightarrow \\
& \Leftrightarrow \frac{m+k}{2 m} \leq \varphi(\alpha)<\frac{m+k+1}{2 m} \Leftrightarrow \varphi^{-1}\left(\frac{m+k}{2 m}\right) \leq \alpha<\varphi^{-1}\left(\frac{m+k+1}{2 m}\right) .
\end{aligned}
$$

Remark 13. By Theorem 1, simple majority, $M_{0}$, and unanimous majority, $M_{m-1}$, can be obtained by $\alpha$-DAF associated with quasiarithmetic means. So, if $\varphi:[0,1] \longrightarrow[0,1]$ is a neutral order automorphism and $\alpha \in\left[\frac{1}{2}, 1\right)$, then the following conditions hold:

1. $M_{0}=F_{\alpha}^{\varphi} \Leftrightarrow \frac{1}{2} \leq \alpha<\varphi^{-1}\left(\frac{m+1}{2 m}\right)$.

2. $M_{m-1}=F_{\alpha}^{\varphi} \Leftrightarrow \varphi^{-1}\left(\frac{2 m-1}{2 m}\right) \leq \alpha<1$.

We also note that if we work on $\mathcal{S}$ it is easy to check that simple majority is only obtained for $\alpha=\frac{1}{2}$.

In the following theorem we justify that for all $\alpha \in\left(\frac{1}{2}, 1\right)$, every $M_{k}$ majority can be obtained by the $\alpha$-DAF associated with some neutral quasiarithmetic mean. So, it is possible to extend every $M_{k}$ majority to fuzzy preferences by means of quasiarithmetic means associated with neutral order automorphisms. However, this extension is not unique. We note that for $\alpha=\frac{1}{2}$, by Remark 15, we have $M_{0}=F_{\frac{1}{2}}^{\varphi}$, for every neutral order automorphism $\varphi$. 
Theorem 2. For every $k \in\{0, \ldots, m-1\}$ and every $\alpha \in\left(\frac{1}{2}, 1\right)$, there exists a neutral order automorphism $\varphi:[0,1] \longrightarrow[0,1]$ such that $M_{k}=F_{\alpha}^{\varphi}$.

Proof. By Theorem 1, we have

$$
M_{k}=F_{\alpha}^{\varphi} \Leftrightarrow \frac{m+k}{2 m} \leq \varphi(\alpha)<\frac{m+k+1}{2 m} .
$$

Now we take $\psi:\left[\frac{1}{2}, 1\right] \longrightarrow\left[\frac{1}{2}, 1\right]$, defined by

$$
\psi(a)= \begin{cases}\frac{1}{2}+\frac{2 k+1}{4 m\left(\alpha-\frac{1}{2}\right)}\left(a-\frac{1}{2}\right), & \text { if } a \in\left[\frac{1}{2}, \alpha\right], \\ 1+\frac{2 m-2 k-1}{4 m(1-\alpha)}(a-1), & \text { if } a \in(\alpha, 1] .\end{cases}
$$

Then we have $\psi\left(\frac{1}{2}\right)=\frac{1}{2}, \psi(1)=1$ and

$$
\psi(\alpha)=\frac{2 m+2 k+1}{4 m} \in\left(\frac{m+k}{2 m}, \frac{m+k+1}{2 m}\right) .
$$

Thus, the mapping $\varphi:[0,1] \longrightarrow[0,1]$, defined by

$$
\varphi(a)= \begin{cases}1-\psi(1-a), & \text { if } a \in\left[0, \frac{1}{2}\right), \\ \psi(a), & \text { if } a \in\left[\frac{1}{2}, 1\right]\end{cases}
$$

is a neutral order automorphism that satisfies the required condition.

\section{OWA operators}

In this section we introduce the OWA operators, a class of aggregation functions where the collective intensity of preference is defined as a weighted average, not of the individual intensities of preference, but of their ordered values. Moreover, we prove that $M_{k}^{l}$ majorities are generated through the class of window OWA operators.

Definition 10. Given $\mathbf{w}=\left(w_{1}, \ldots, w_{m}\right) \in[0,1]^{m}$ satisfying $\sum_{i=1}^{m} w_{i}=1$, the OWA operator associated with $\mathbf{w}$ is the aggregation function $F^{\mathbf{w}}$ defined by

$$
F^{\mathbf{w}}\left(r_{1}, \ldots, r_{m}\right)=\sum_{i=1}^{m} w_{i} \cdot r_{\sigma(i)},
$$

where $\sigma$ is a permutation of $\{1, \ldots, m\}$ such that $r_{\sigma(1)} \geq \cdots \geq r_{\sigma(m)}$. 
Simple examples of OWA operators are

$$
F^{\mathbf{w}}\left(r_{1}, \ldots, r_{m}\right)= \begin{cases}\max \left(r_{1}, \ldots, r_{m}\right), & \text { when } \mathbf{w}=(1,0, \ldots, 0), \\ \min \left(r_{1}, \ldots, r_{m}\right), & \text { when } \mathbf{w}=(0, \ldots, 0,1), \\ \frac{r_{1}+\cdots+r_{m}}{m}, & \text { when } \mathbf{w}=\left(\frac{1}{m}, \frac{1}{m}, \ldots, \frac{1}{m}\right) .\end{cases}
$$

Now we establish that every OWA operator is anonymous, monotonic and unanimous. Moreover, we give a necessary and sufficient condition so that it will be neutral.

Proposition 5. For every $\mathbf{w}=\left(w_{1}, \ldots, w_{m}\right) \in[0,1]^{m}$ such that $\sum_{i=1}^{m} w_{i}=1$ the following statements hold:

1. $F^{\mathrm{w}}$ is anonymous, monotonic and unanimous.

2. $F^{\mathbf{w}}$ is neutral $\Leftrightarrow w_{m+1-i}=w_{i}$ for all $i \in\left\{1, \ldots,\left[\frac{m}{2}\right]\right\}$.

Proof.

1. Obvious.

2. Given $\left(r_{1}, \ldots, r_{m}\right) \in[0,1]^{m}$, let $\sigma$ be a permutation of $\{1, \ldots, m\}$ such that $r_{\sigma(1)} \geq \cdots \geq$ $r_{\sigma(m)}$. Therefore, we have $1-r_{\sigma(m)} \geq \cdots \geq 1-r_{\sigma(1)}$. Thus,

$$
\begin{aligned}
& F^{\mathbf{w}}\left(1-r_{1}, \ldots, 1-r_{m}\right)=1-F^{\mathbf{w}}\left(r_{1}, \ldots, r_{m}\right) \Leftrightarrow \\
& \Leftrightarrow \sum_{i=1}^{m} w_{i} \cdot\left(1-r_{\sigma(m+1-i)}\right)=1-\sum_{i=1}^{m} w_{i} \cdot r_{\sigma(i)} \Leftrightarrow \\
& \Leftrightarrow \sum_{i=1}^{m} w_{i} \cdot r_{\sigma(m+1-i)}=\sum_{i=1}^{m} w_{i} \cdot r_{\sigma(i)} \Leftrightarrow \\
& \Leftrightarrow \sum_{i=1}^{m} w_{m+1-i} \cdot r_{\sigma(i)}=\sum_{i=1}^{m} w_{i} \cdot r_{\sigma(i)} .
\end{aligned}
$$

The last equality is true for all $\left(r_{1}, \ldots, r_{m}\right) \in[0,1]^{m}$ if and only if $w_{m+1-i}=w_{i}$ for all $i \in\left\{1, \ldots,\left[\frac{m}{2}\right]\right\}$.

Now the window OWA operators are defined. They average only the central values, and do not consider the extreme values. 
Definition 11. Given $j \in\left\{1, \ldots,\left[\frac{m+1}{2}\right]\right\}$, we define $\mathbf{w}^{j}=\left(w_{1}^{j}, \ldots, w_{m}^{j}\right)$ as

$$
w_{i}^{j}= \begin{cases}\frac{1}{m-2(j-1)}, & \text { if } i=j, j+1, \ldots, m-(j-1), \\ 0, & \text { otherwise. }\end{cases}
$$

The OWA operator associated with $\mathbf{w}^{j}, F^{\mathbf{w}^{j}}$, called $j$-th window OWA operator, is defined by

$$
F^{\mathbf{w}^{j}}\left(r_{1}, \ldots, r_{m}\right)=\frac{r_{\sigma(j)}+\cdots+r_{\sigma(m-(j-1))}}{m-2(j-1)} .
$$

Obviously, it is neutral (and by Proposition 3, it is also anonymous, monotonic and unanimous).

Remark 14. $F^{\mathbf{w}^{1}}$ is the arithmetic mean. $F^{\mathbf{w}^{2}}$ makes the average of the individual preferences excepting the minimum and the maximum values. $F^{\mathbf{w}^{3}}$ makes the average of the individual preferences excepting the two minimum and the two maximum values. Finally, $F^{\mathbf{w}^{\left[\frac{m+1}{2}\right]}}$ makes the average of the two central values of the individual preferences, if $m$ is even; and it selects the central value of the individual preferences, if $m$ is odd.

Remark 15. The restriction of the $j$-th window OWA operator, $F^{\mathbf{w}^{j}}$, to profiles of crisp preferences $\mathbf{r} \in\left\{0, \frac{1}{2}, 1\right\}^{m}$, is represented by the mapping $f^{\mathbf{w}^{j}}: \mathcal{M} \longrightarrow[0,1]$ defined by

$$
f^{\mathbf{w}^{j}}\left(m_{1}, m_{2}, m_{3}\right)=\sum_{i=1}^{m_{1}} w_{i}^{j}+\frac{1}{2} \sum_{i=1}^{m_{2}} w_{m_{1}+i}^{j} .
$$

According to six possible cases of $m_{1}, m_{2}$ and $m_{3}, f^{\mathbf{w}^{j}}$ takes the following values:

1. If $m_{1}<j$ and $m_{1}+m_{2}<j$, then $f^{\mathbf{w}^{j}}\left(m_{1}, m_{2}, m_{3}\right)=0$.

2. If $m_{1}<j$ and $j \leq m_{1}+m_{2} \leq m-(j-1)$, then

$$
f^{\mathbf{w}^{j}}\left(m_{1}, m_{2}, m_{3}\right)=\frac{1}{2} \frac{m_{1}+m_{2}-(j-1)}{m-2(j-1)} .
$$

3. If $m_{1}<j$ and $m_{1}+m_{2}>m-(j-1)$, then $f^{\mathbf{w}^{j}}\left(m_{1}, m_{2}, m_{3}\right)=\frac{1}{2}$.

4. If $j \leq m_{1} \leq m-(j-1)$ and $j \leq m_{1}+m_{2} \leq m-(j-1)$, then

$$
f^{\mathbf{w}^{j}}\left(m_{1}, m_{2}, m_{3}\right)=\frac{m_{1}-(j-1)}{m-2(j-1)}+\frac{1}{2} \frac{m_{2}}{m-2(j-1)} .
$$

5. If $j \leq m_{1} \leq m-(j-1)$ and $m_{1}+m_{2}>m-(j-1)$, then 


$$
f^{\mathbf{w}^{j}}\left(m_{1}, m_{2}, m_{3}\right)=\frac{m_{1}-(j-1)}{m-2(j-1)}+\frac{1}{2} \frac{m-(j-1)-m_{1}}{m-2(j-1)} .
$$

6. If $m_{1}>m-(j-1)$ and $m_{1}+m_{2}>m-(j-1)$, then $f^{\mathbf{w}^{j}}\left(m_{1}, m_{2}, m_{3}\right)=1$.

Now we establish that every $\alpha$-DAF associated with a window OWA operator is a $M_{k}^{l}$ majority or, in some particular cases, a $M_{k}$ majority or the absolute majority.

Proposition 6. If $j \in\left\{1, \ldots,\left[\frac{m+1}{2}\right]\right\}$ and $\alpha \in\left[\frac{1}{2}, 1\right)$, then $F_{\alpha}^{\mathbf{w}^{j}}$ coincides with:

1. $M_{[(2 \alpha-1) m]}$, if $j=1$.

2. The absolute majority, if $m$ is odd and $j=\frac{m+1}{2}$.

3. $M_{k}^{l}$, where $k=[(2 \alpha-1)(m-2(j-1))]$ and $l=j-1+k$, if $j \in\left\{2, \ldots,\left[\frac{m}{2}\right]\right\}$.

Proof. We note that for every $\left(m_{1}, m_{2}, m_{3}\right) \in \mathcal{M}$ we have:

$$
f_{\alpha}^{\mathbf{w}^{j}}\left(m_{1}, m_{2}, m_{3}\right)=1 \Leftrightarrow f^{\mathbf{w}^{j}}\left(m_{1}, m_{2}, m_{3}\right)>\alpha .
$$

According to Remark 15, $f^{\mathbf{w}^{j}}\left(m_{1}, m_{2}, m_{3}\right)>\alpha$ is not possible in the cases 1, 2 and 3 ; and it is always satisfied in the case 6 . So, we need to study only the cases 4 and 5 , where $j \leq m_{1} \leq m-(j-1)$ holds. In case $4 m_{2} \leq m-(j-1)-m_{1}$ holds; while in case 5 we have $m_{2}>m-(j-1)-m_{1}$. So then, in cases 4 and 5 we have

$$
\begin{aligned}
& f^{\mathbf{w}^{j}}\left(m_{1}, m_{2}, m_{3}\right)=\frac{m_{1}-(j-1)}{m-2(j-1)}+\frac{1}{2} \frac{\min \left(m_{2}, m-(j-1)-m_{1}\right)}{m-2(j-1)}= \\
& =\frac{m_{1}-(j-1)}{m-2(j-1)}+\frac{m-m_{1}+\min \left(-m_{3},-(j-1)\right)}{2(m-2(j-1))}= \\
& =\frac{m_{1}-(j-1)}{m-2(j-1)}+\frac{m-m_{1}-\max \left(m_{3}, j-1\right)}{2(m-2(j-1))} .
\end{aligned}
$$

Consequently, in these cases we have

$$
\begin{aligned}
& f^{\mathbf{w}^{j}}\left(m_{1}, m_{2}, m_{3}\right)>\alpha \Leftrightarrow \\
& \Leftrightarrow \frac{m_{1}-(j-1)}{m-2(j-1)}+\frac{m-m_{1}-\max \left(m_{3}, j-1\right)}{2(m-2(j-1))}>\alpha \Leftrightarrow
\end{aligned}
$$




$$
\begin{aligned}
& \Leftrightarrow 2 m_{1}-2(j-1)+m-m_{1}-\max \left(m_{3}, j-1\right)>2 \alpha(m-2(j-1)) \Leftrightarrow \\
& \Leftrightarrow m_{1}>\max \left(m_{3}, j-1\right)+(2 \alpha-1)(m-2(j-1)) .
\end{aligned}
$$

Therefore, for every $\left(m_{1}, m_{2}, m_{3}\right) \in \mathcal{M}$ we have

$$
\begin{aligned}
& f^{\mathbf{w}^{j}}\left(m_{1}, m_{2}, m_{3}\right)>\alpha \Leftrightarrow \\
& \Leftrightarrow\left\{\begin{array}{l}
m_{1}>m-(j-1) \\
\text { or } \\
j \leq m_{1} \leq m-(j-1) \quad \text { and } \quad m_{1}>\max \left(m_{3}, j-1\right)+(2 \alpha-1)(m-2(j-1))
\end{array}\right.
\end{aligned}
$$

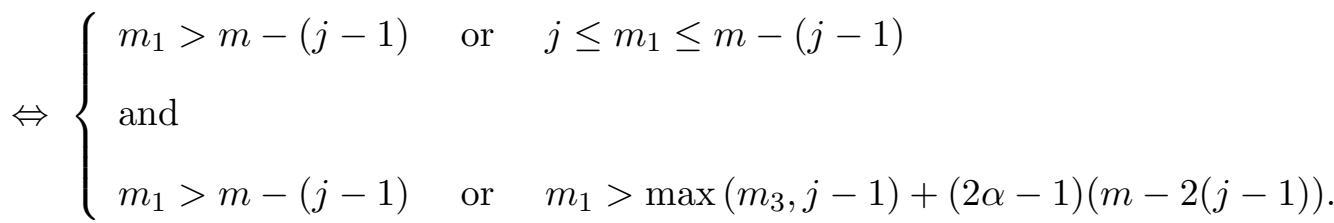

We note that if $m_{1}>m-(j-1)$, since $m_{3}<j-1$ and

$$
j-1+(2 \alpha-1)(m-2(j-1))<m-(j-1),
$$

for all $\alpha \in\left[\frac{1}{2}, 1\right)$, we have

$$
m_{1}>\max \left(m_{3}, j-1\right)+(2 \alpha-1)(m-2(j-1)) .
$$

Hence,

$$
\begin{aligned}
& f^{\mathbf{w}^{j}}\left(m_{1}, m_{2}, m_{3}\right)>\alpha \Leftrightarrow \\
& \Leftrightarrow\left\{\begin{array}{l}
m_{1} \geq j \\
\text { and } \\
m_{1}>\max \left(m_{3}, j-1\right)+(2 \alpha-1)(m-2(j-1))
\end{array}\right. \\
& \Leftrightarrow m_{1}>\max \left(m_{3}, j-1\right)+(2 \alpha-1)(m-2(j-1)) \\
& \Leftrightarrow m_{1}>\max \left(m_{3}, j-1\right)+[(2 \alpha-1)(m-2(j-1))] .
\end{aligned}
$$

Consequently,

$$
f_{\alpha}^{\mathbf{w}^{j}}\left(m_{1}, m_{2}, m_{3}\right)=1 \Leftrightarrow m_{1}>\max \left(m_{3}, j-1\right)+[(2 \alpha-1)(m-2(j-1))] .
$$


Now we justify the required conditions, according to the different values of $m$ and $j$.

1. If $j=1$, then

$$
f_{\alpha}^{\mathbf{w}^{1}}\left(m_{1}, m_{2}, m_{3}\right)=1 \Leftrightarrow m_{1}>m_{3}+[(2 \alpha-1) m] .
$$

So, $F_{\alpha}^{\mathbf{w}^{1}}=M_{[(2 \alpha-1) m]}$.

2. If $m$ is odd and $j=\frac{m+1}{2}$, then

$$
\begin{aligned}
& f_{\alpha}^{\mathbf{w}^{j}}\left(m_{1}, m_{2}, m_{3}\right)=1 \Leftrightarrow m_{1}>\max \left(m_{3}, \frac{m-1}{2}\right)+[2 \alpha-1] \Leftrightarrow \\
& \Leftrightarrow m_{1}>\max \left(m_{3}, \frac{m-1}{2}\right) .
\end{aligned}
$$

By $m_{1}+m_{3} \leq m$, if $m_{1}>\frac{m-1}{2}$, then $m_{3}<m-\frac{m-1}{2}=\frac{m+1}{2}$, i.e., $m_{3} \leq \frac{m-1}{2}$. Thus,

$$
f_{\alpha}^{\mathbf{w}^{j}}\left(m_{1}, m_{2}, m_{3}\right)=1 \Leftrightarrow m_{1}>\max \left(m_{3}, \frac{m-1}{2}\right) \Leftrightarrow m_{1}>\frac{m-1}{2} \Leftrightarrow m_{1}>\frac{m}{2} .
$$

So, $F_{\alpha}^{\mathbf{w}^{j}}$ coincides with the absolute majority.

3. Let $j \in\left\{2, \ldots,\left[\frac{m}{2}\right]\right\}, k=[(2 \alpha-1)(m-2(j-1))]$ and $l=j-1+k$. We have

$$
f_{\alpha}^{\mathbf{w}^{j}}\left(m_{1}, m_{2}, m_{3}\right)=1 \Leftrightarrow m_{1}>\max \left(m_{3}, l-k\right)+k .
$$

Since

$$
\max \left(m_{3}, l-k\right)+k=\max \left(m_{3}+k, l\right),
$$

we obtain $F_{\alpha}^{\mathbf{w}^{j}}=M_{k}^{l}$.

Remark 16. We have noted that $F^{\mathbf{w}^{1}}$ and $F^{\varphi}$, when $\varphi$ is the identity automorphism, are the arithmetic mean. Considering these cases in Propositions 4 and 6, we obtain the same result: the $\alpha-\mathrm{DAF}$ associated with the arithmetic mean coincides with $M_{[(2 \alpha-1) m]}$.

In the following theorem we justify that for every $M_{k}^{l}$ majority, there exist a window OWA operator and some $\alpha \in\left[\frac{1}{2}, 1\right)$ such that $M_{k}^{l}$ is the $\alpha$-DAF associated with the window OWA operator. This result allows to extend every $M_{k}^{l}$ majority to fuzzy preferences by means of window OWA operators.

Theorem 3. If $j \in\left\{2, \ldots,\left[\frac{m}{2}\right]\right\}, k \in\{0,1, \ldots, m-4\}, l \in\left\{k+1, \ldots,\left[\frac{m+k}{2}\right]-1\right\}$ and $\alpha \in\left[\frac{1}{2}, 1\right)$, then:

$$
M_{k}^{l}=F_{\alpha}^{\mathbf{w}^{j}} \Leftrightarrow\left\{\begin{array}{l}
j=l-k+1 \\
\text { and } \\
\frac{1}{2}+\frac{k}{2(m-2(l-k))} \leq \alpha<\frac{1}{2}+\frac{k+1}{2(m-2(l-k))} .
\end{array}\right.
$$


Proof. By Proposition 6 and Remark 10, we have

$$
M_{k}^{l}=F_{\alpha}^{\mathbf{w}^{j}} \Leftrightarrow\left\{\begin{array}{l}
l=j-1+k \\
\text { and } \\
k=[(2 \alpha-1)(m-2(j-1))] .
\end{array}\right.
$$

Then, we only need to prove

$$
k=[(2 \alpha-1)(m-2(j-1))] \Leftrightarrow \frac{1}{2}+\frac{k}{2(m-2(j-1))} \leq \alpha<\frac{1}{2}+\frac{k+1}{2(m-2(j-1))} .
$$

Since $j \in\left\{2, \ldots,\left[\frac{m}{2}\right]\right\}$, we have $j<\frac{m+2}{2}$ and, consequently, $m-2(j-1)>0$. Hence

$$
\begin{aligned}
& k=[(2 \alpha-1)(m-2(j-1))] \Leftrightarrow k \leq(2 \alpha-1)(m-2(j-1))<k+1 \Leftrightarrow \\
& \Leftrightarrow \frac{k}{m-2(j-1)} \leq 2 \alpha-1<\frac{k+1}{m-2(j-1)} \Leftrightarrow \\
& \Leftrightarrow \frac{1}{2}+\frac{k}{2(m-2(j-1))} \leq \alpha<\frac{1}{2}+\frac{k+1}{2(m-2(j-1))} . \quad \text { - }
\end{aligned}
$$




\section{References}

Bezdek, J.C., Spillman, B., Spillman, R., 1978. A fuzzy relation space for group decision theory. Fuzzy Sets and Systems 1, 255-268.

Bullen, P.S., Mitrinović, D.S., Vasić, P.M., 1988. Means and Their Inequalities (Reidel, Dordrecht).

Dubois, D., Prade, H., 1985. A review of fuzzy set aggregation connectives. Information Sciences 36, $85-121$.

Ferejohn, J.A., Grether, D.M., 1974. On a class of rational social decisions procedures. Journal of Economic Theory 8, 471-482.

Fishburn, P.C., 1973. The Theory of Social Choice (Princeton University Press, Princeton).

Fodor, J., Roubens, M., 1994. Fuzzy Preference Modelling and Multicriteria Decision Support (Kluwer Academic Publishers, Dordrecht).

García-Lapresta, J.L., Llamazares, B., 2000. Aggregation of fuzzy preferences: Some rules of the mean. Social Choice and Welfare 17, 673-690.

Grabisch, M., 1996. The application of fuzzy integrals in multicriteria decision making. European Journal of Operational Research 89, 445-456.

Kolmogoroff, A., 1930. Sur la notion de la moyenne. Atti della R. Academia Nazionale del Lincei. Rendiconti della Classe di Scienze Fisiche, Mathematiche e Naturali (6) 12, 388-391.

Marchant, T., 1996. Valued relations aggregation with the Borda method. Journal of Multi-criteria Decision Analysis 5, 127-132.

Marchant, T., 2000. Does the Borda rule provide more than a ranking? Social Choice and Welfare $17,381-391$. 
May, K.O., 1952. A set of independent necessary and sufficient conditions for simple majority decision. Econometrica 20, 680-684.

Nagumo, M., 1930. ber eine Klasse der Mittelwerte. Japanese Journal of Mathematics 7, 71-79.

Nakamura, K., 1992. On the nature of intransitivity in human preferential judgements, in V. Novák, J. Ramík et al., eds., Fuzzy Approach to Reasoning and Decision-Making (Kluwer Academic Publishers, Dordrecht), 147-162.

Nurmi, H., 1981. Approaches to collective decision making with fuzzy preference relations. Fuzzy Sets and Systems 6, 249-259.

Ovchinnikov, S.V., 1990. Means and social welfare function in fuzzy binary relation spaces, in J. Kacprzyk and M. Fedrizzi, eds., Multiperson Decision Making Using Fuzzy Sets and Possibility Theory (Kluwer Academic Publishers, Dordrecht), 143-154.

Saari, D.G., 1990. Consistency of decision processes. Annals of Operations Research 23, 103-137.

Saari, D.G., 1994. Geometry of Voting (Springer-Verlag, Berlin).

Tanino, T., 1984. Fuzzy preference orderings in group decision making. Fuzzy Sets and Systems 12, $117-131$.

Yager, R.R., 1988. Ordered weighted averaging operators in multicriteria decision making. IEEE Transactions on Systems, Man and Cybernetics 8, 183-190.

Yager, R.R., 1991. Connectives and quantifiers in fuzzy sets. Fuzzy Sets and Systems 40, 39-75.

Yager, R.R., Kacprzyk, J., eds., 1997. The Ordered Weighted Averaging Operators: Theory and Applications (Kluwer Academic Publishers, Dordrecht). 